titres. Our patient meets the 2015 Consensus Diagnostic Criteria for NMOSD. ${ }^{1}$ Treatment with high dose corticosteroids and rituximab lead to clinical and radiological improvement, but she had a clinical relapse 10 months later with new LETM (T3-T7), necessitating increased immunosuppression with more rigorous rituximab dosing of $1000 \mathrm{mg}$ every 6 months.

Conclusions Double positivity for both Aquaporin-4 and MOG antibodies in NMOSD is rare. We describe a case of doublepositive NMOSD occurring following an infective illness. This case demonstrates that NMOSD may occasionally masquerade as post-infectious Acute Disseminated Encephalomyelitis and highlights the importance of checking antibodies in these patients, given the treatment strategies and risk of relapse differs considerably.

\section{ANTI-LEUCINE-RICH GLIOMA INACTIVATED 1 (LGI1) ENCEPHALITIS ASSOCIATED WITH HIGH GRADE PAPILLARY UROTHELIAL CARCINOMA}

Jessica Stabler, David Williams. John Hunter Hospital, Newcastle West, NSW, Australia

\subsection{6/bmjno-2021-ANZAN.105}

Introduction LGI1 encephalitis is a rare form of limbic encephalitis, that was first recognised as a primary autoimmune phenomenon, and subsequently described in association with a limited number of malignancies. ${ }^{1}$ We report a novel case of LGI-1 encephalitis occurring concurrent to a high-grade papillary urothelial carcinoma.

Case Presentation A previously well 72-year-old male presented to a rural hospital with a first episode generalised tonic-clonic seizure, confusion and progressive behavioural change. He was diagnosed with LGI-1 encephalitis, with positive CSF antibodies, and mesial temporal T2 hyperintensity on MRI brain. There was no response to first line treatment with steroids, intravenous immunoglobulin, and mycophenolate. Malignancy screening revealed a lesion within the upper pole of the left kidney, favoured to represent a transitional cell carcinoma. Biopsy demonstrated a low grade papillary urothelial carcinoma. The patient's encephalopathy continued to worsen over a period of months, despite ongoing immunosuppression. He underwent a left nephrouretectomy, and histology demonstrated a high-grade papillary urothelial carcinoma. Subsequent to this, there was improvement in cognition and behaviour. Psychotropic and immunosuppressive medications were slowly weaned. At 9month follow-up, the patient has returned close to baseline function, and has been clinically stable off all immunosuppressive treatment.

Conclusions LGI-1 encephalitis has previously been described in association with thymoma, lymphoma, teratoma, and more recently with lung and prostate cancer. ${ }^{2}{ }^{3}$ We believe our case is the first report of association between LGI-1 encephalitis and high grade papillary urothelial carcinoma.

\section{REFERENCES}

1. van Sonderen A, et al. Anti- LGI1 encephalitis. Neurology Oct 2016;87 (14):1449-1456.

2. Navalli D, Mutalik NR, Jayalakshmi G. Leucine-rich glioma-inactivated protein antibody-positive limbic encephalitis in a patient with adenocarcinoma of prostate: a case report. Ann Indian Acad Neurol Jan-Mar 2019:22(1):121-122.

3. Virupakshaiah A, Dalakas M, Desai N, Mintzer S, Ratliff J. A report on LGI1 encephalitis in association with squamous cell carcinoma of the lung. Neurology Apr 2019;92(15 Supp):2.2-028.

\section{CONTRAST-INDUCED ENCEPHALOPATHY AFTER CARBON DIOXIDE ANGIOGRAPHY IN THE UPPER EXTREMITY AND IODINATED CONTRAST - A CASE REPORT}

${ }^{1,2}$ Hue Mun Au Yong, ${ }^{3}$ Matthew K Ligtermoet, ${ }^{3}$ Douglas Crompton. ${ }^{1}$ Department of Neurology, Alfred Health, Melbourne, VIC, Australia; ${ }^{2}$ Department of Neurology, Royal Melbourne Hospital, Parkville, VIC, Australia; ${ }^{3}$ Department of Neurology, Northern Health, Epping, VIC, Australia

\subsection{6/bmjno-2021-ANZAN.106}

Objective Carbon dioxide $\left(\mathrm{CO}_{2}\right)$ is used as an alternative contrast agent in angiography for patients with iodinated contrast allergy or impaired renal function. $\mathrm{CO}_{2}$ angiography is contraindicated in cerebral circulation based on demonstrated neurotoxicity in animals. ${ }^{1} 2$ We present a case of reversible neurological complications post $\mathrm{CO}_{2}$ angiography and iodinated contrast.

Methods and Results A 65-year-old man presented with an ischaemic finger from steal syndrome post-arteriovenous fistula ligation, on a background of end-stage renal disease and type 2 diabetes. He underwent $\mathrm{CO}_{2}$ angiography for evaluation of right upper limb circulation. Immediately after the first $\mathrm{CO}_{2}$ injection into the right brachial artery, he became unresponsive and spontaneously recovered after 1-minute. Due to poor distal opacification with $\mathrm{CO}_{2}, 15 \mathrm{ml}$ of iodinated contrast was administered. At 3-hour post-procedure, he developed left facial droop, left hemiparesis and left visual neglect. CT brain, angiogram and perfusion study at 5-hour post-procedure showed no acute changes. Overnight, he worsened to dense left hemiplegia. Non-contrast CT brain at 11-hour post-procedure showed oedema and hyperdensity in the right hemisphere. He had a seizure on day 1 post-procedure. MRI brain performed 24-hour post-procedure showed dramatic resolution of right hemispheric cerebral oedema with no diffusion restriction. All neurological deficits completely resolved 7-day postprocedure.

The $\mathrm{CO}_{2}$ which refluxed into the cerebral circulation from the brachial artery, caused the breakdown of blood-brain barrier, allowing penetration of iodinated contrast and subsequent right hemispheric cerebral oedema.

Conclusions This case highlights the risk of air embolism and neurotoxicity of $\mathrm{CO}_{2}$ angiography and the rare occurrence of contrast-induced encephalopathy.

\section{REFERENCES}

1. Coffey R, Quisling RG, Mickle JP, et al. The cerebrovascular effects of intraarterial CO2 in quantities required for diagnostic imaging. Radiology 1984;151(2):40510. doi:10.1148/radiology.151.2.6424174

2. Kozlov DB, Lang EV, Barnhart W, et al. Adverse cerebrovascular effects of intraarterial CO2 injections: development of an in vitro/in vivo model for assessment of gas-based toxicity. Journal of Vascular and Interventional Radiology: JVIR 2005;16(5):713-26. doi: 10.1097/01.Rvi.0000153114.05700.61 [published Online First: 2005/05/06]

\section{A TAIL OF TWO NMDA RECEPTOR ANTIBODY ENCEPHALITIDIES: AGGRESSIVE TREATMENT, DIVERGENT OUTCOMES}

${ }^{1}$ Christopher Blair, ${ }^{2,3}$ Nicolas Urriola, 'Leon Edwards, ${ }^{1}$ Patrick Aouad. ${ }^{1}$ Department of Neurology and Neurophysiology, Liverpool Hospital, Liverpool, NSW, Australia; ${ }^{2}$ Department of Immunology, Liverpool Hospital, Liverpool, NSW, Australia; ${ }^{3}$ Department of Immunology, Royal Prince Alfred Hospital, Camperdown, NSW, Australia

10.1136/bmjno-2021-ANZAN.107 